\title{
A Novel MicroPhotonic Structure for Optical Header Recognition
}

\author{
Muhsen Aljada ${ }^{1}$, Kamal Alameh ${ }^{1}$, Adam Osseiran ${ }^{2}$, and Khalid Al-Begain ${ }^{3}$ \\ ${ }^{1}$ Centre for MicroPhotonic Systems, ${ }^{2}$ National Networked TeleTest \\ Facility, Electron Science Research Institute, Edith Cowan University, \\ Joondalup, WA 6027, Australia \\ WWW home page: http://comps.ecu.edu.au \\ ${ }^{3}$ Mobile Computing, Communications and Networking Research Group, \\ School of Computing, University of Glamorgan, Pontypridd (Cardiff), \\ CF37 1DL, Wales, UK.
}

\begin{abstract}
In this paper, we propose and demonstrate a new MicroPhotonic structure for optical packet header recognition based on the integration of an optical cavity, optical components and a photoreceiver array. The structure is inherently immune to optical interference thereby routing an optical header within optical cavities to different photo receiver elements to generate the autocorrelation function, and hence the recognition of the header using simple microelectronic circuits. The proof-of-concept of the proposed MicroPhotonic optical header recognition structure is analysed and experimentally demonstrated, and results show excellent agreement between measurements and theory.
\end{abstract}

\section{Introduction}

The rapid and global spread of the internet is accelerating the growth of optical communication networks and the demand for more bandwidth has driven the use of photonic technology in telecommunication and computer networks. The diversity of future services will require high-capacity optical networks featuring dynamic and high-speed routing and switching of data packets [1], [2].

The new generation very high-bit rate optical packet switched networks require a potentially faster approach to decode the header bits optically so that a given routing decision can be made on-the-fly. Currently, to make routing decisions, optical packets are converted into the electrical domain and electronic signal processing is used to recognize the optical headers, as shown in Fig. 1 [3]. This approach cannot 
handle the high data rates of future generation packet switched optical networks, and this issue is currently the bottleneck for recognizing high-speed optical headers in future optical networks [4].

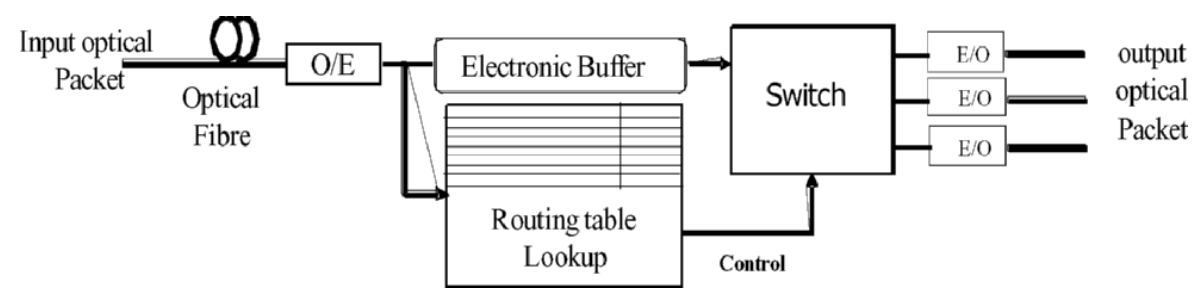

Fig. 1. Conventional optical packet switching node

Correlation is an important signal-processing function that is commonly used to recognize an incoming pattern by comparing it with a predetermined pattern. At the appropriate sample time, a high-amplitude lobe is produced if the input pattern is an exact match to the predetermined pattern. Optical Header recognition based on optical correlation is a promising concept to perform the header pattern optically by using time-domain correlator to match it to a lookup table constructed using a bank of optical correlators, as illustrated in Fig. 2. The stream of information from source to destination usually consists of small optical packets; each comprises a header and a payload. An optical tap is used to bypass a small power of the optical packets. This small power is uniformly split using the $1 \times \mathrm{M}$ optical splitter, and distributed into $\mathrm{M}$ optical correlators of different predetermined patterns. Each correlator is assigned a single destination address and designed to generate an electrical waveform that represents the correlation function between its destination address and the present optical header. By sampling the various correlation signals and comparing them, using comparators, to threshold levels, only one autocorrelation function is generated, which corresponds to the correlator whose destination address matches the optical header pattern. Control signals are generated and fed into the control ports of the N-port optical switch that routes the transmitted packets to their next hops [5].

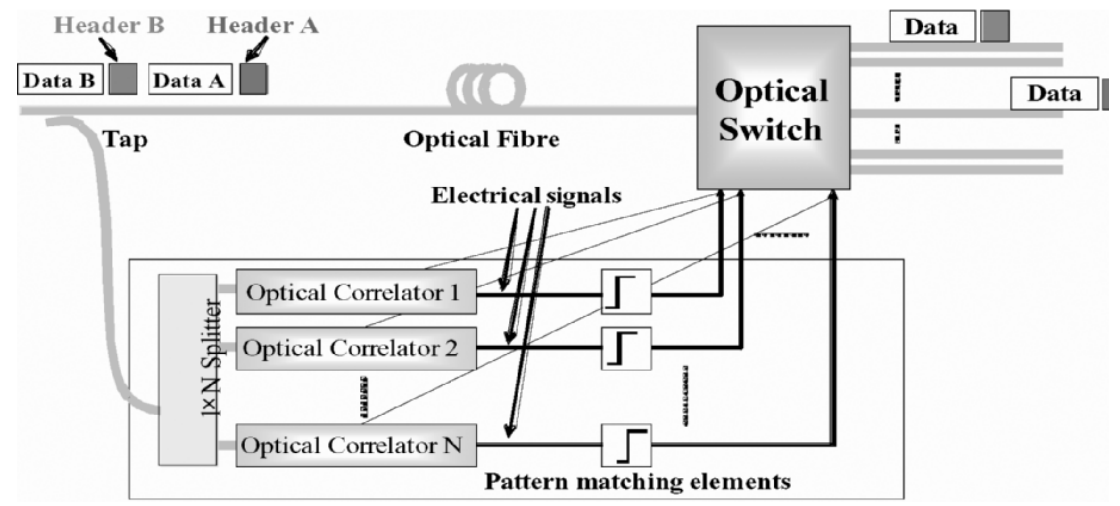

Fig. 2. Generic concept of optical header recognition using optical correlators 
Fibre-based optical correlation techniques have been investigated for several years for their potential to recognize high-speed incoming bit streams, with essentially no latency. Numerous optical correlator designs have been proposed and experimentally demonstrated including the use of fibre matched filter [6],[7], spectro-holographic filter [8], optical AND gates [9],[10], loop mirror configurations with semiconductor optical amplifier [11], integrated optical chip [12], optical serial to parallel conversion [13-15], time stretching technique [16], and Opto-VLSI processor [17]. These designs suffer from high power requirement and low efficiency. Recently, a set of fibre Bragg gratings (FBG) in conjunction with a single photodetector have been proposed to construct a fibre-based optical correlator for optical header recognition [18-26]. FBG-based correlators have some specific advantages over other designs, however, they are susceptible to optical interference caused by the detection of delayed optical bits using a single photodetector when the coherent time of the optical signal is higher than the bit time delay created by the FBGs. This effect severely limits the stability of the correlation output and hence degrades the service and reliability of the optical network.

In this paper we propose and demonstrate a new structure for header recognition using a new MicroPhotonic structure that integrates an optical cavity, optical components and a VLSI photoreceiver array. The structure is scalable, and inherently immune to optical interference. This paper is organized as follow; in section 2 is the proposed MicroPhotonic optical header recognition structure, experimental setup and results are in section 3, and the paper is concluded with some remarks in section 4 .

\section{MicroPhotonic Optical Correlator Structure}

Fig. 3 schematically illustrates the MicroPhotonic correlator architecture for optical header recognition of the present invention. The small power of the optical packet, which consists of the optical payload and the optical header, is by-passed from the optical fibre using the optical tap (about $10 \%$ is tapped). The $1 \times 1 \mathrm{~N}$ optical splitter equally splits the tapped optical packet into $\mathrm{N}$ packets. The microlenses are appropriately etched into the optical substrate in order to convert the in-fibre optical packets into collimated optical beams. Each collimated optical beam propagates within the optical substrate and undergoes several reflections in a cavity whose width is defined by the mirror and the diffractive optical element (DOE). Every time a beam hits the DOE, a small fraction of its power is transmitted through the DOE for detection and amplification by an element of the wideband photoreceiver array that is integrated on the surface of the optical substrate, while the remaining large fraction is reflected and routed for subsequent delayed photodetection. The amplitude of a received optical signal can be set to a low value (0) or a high value (1) by adjusting the photoreceiver's amplifier gain. Each element of the combiner/comparator array adds the amplified photocurrents of a photoreceiver row and generates an output signal. An autocorrelation function of a very high peak is generated whenever the optical header matches a pattern of the correlator, while for all other patterns, only low intensity cross-correlation functions are produced. Threshold detectors (comparators) are used at the outputs of the optical correlators to 
provide an electrical match/no-match signal to the optical switch that uses these signals to determine to which output port each packet should be forwarded.

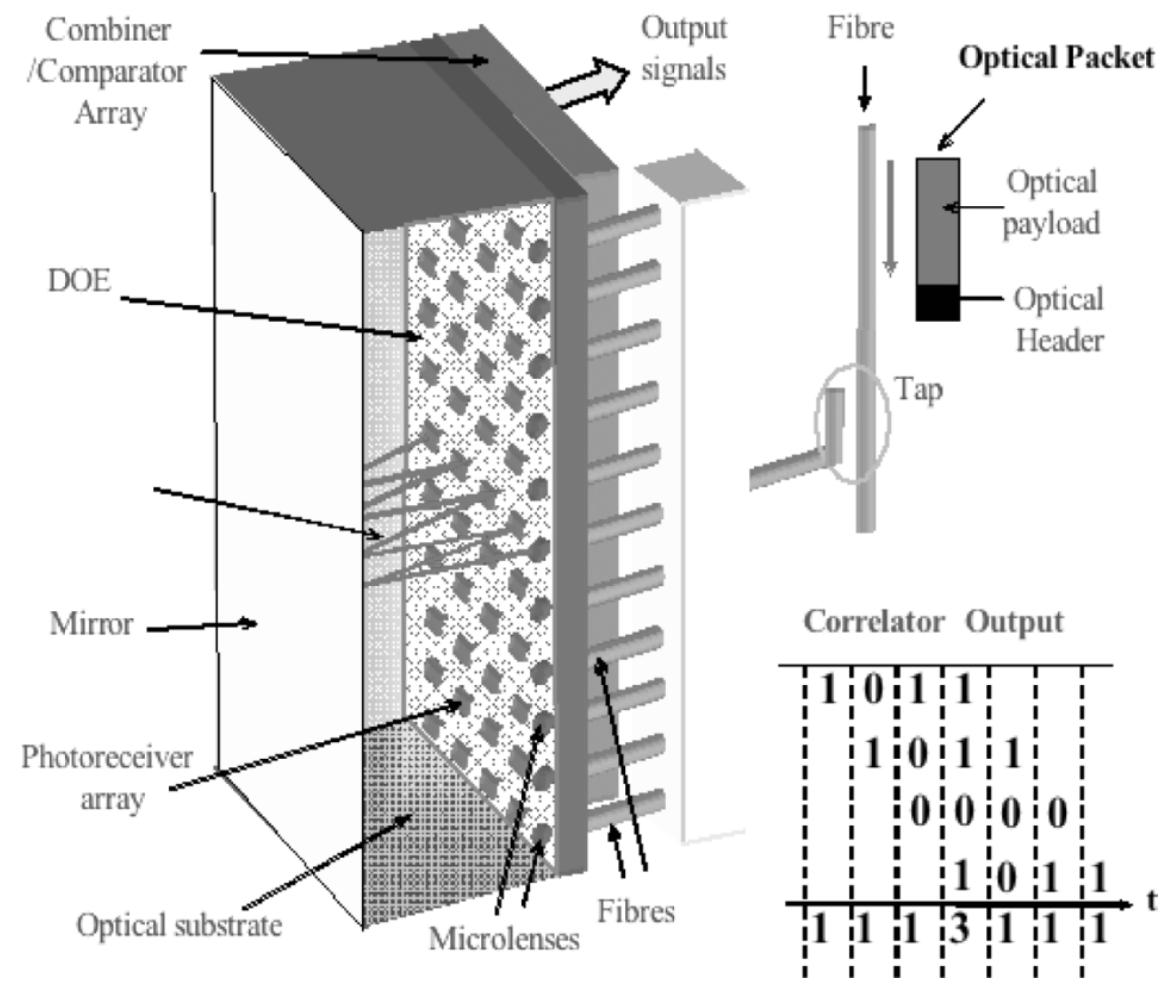

Fig. 3. MicroPhotonic correlator architecture

Fig. 4 schematically illustrates the interface between the photoreceiver array and the optical substrate and also illustrates the propagation of the optical beams inside the optical substrate. The optical packet propagating in the input fibre is converted into a collimated optical beam via the microlens. The glass layer is used over the photoreceiver chip for protection. The DOE is inserted between the photoreceiver chip and the optical substrate. The DOE comprises two sections. The first section is the beam router, which is a hologram capable of steering collimated optical beam, while the second section (dashed) acts as a lens relay that prevents the cavity beam from diverging as it propagates along its optical path, and also maintains its diameter within an adequate range. The DOE can be appropriately coated to provide any desired reflectivity. As the cavity beam hits the DOE, a large portion of its power is reflected inside the optical cavity and its diameter is equalized for subsequent propagation, while a small fraction of its power is transmitted through the DOE and the glass layer and then detected by one of the photoreceivers. For a cavity length $\mathrm{L}$ and a photoreceiver spacing $\mathrm{d}$, the steering angle, $\theta$, of the beam router is $\arctan (\mathrm{d} / 2 \mathrm{~L})$. The output sequence from the correlator is given by: 


$$
y\left(m T_{b i t}\right)=\sum_{j=0}^{n-1} h_{j} \cdot x\left(m T_{b i t}-j T_{b i t}\right)
$$

where $n$ is the number of bits in the header sequence, $T_{b i t}$ is the bit time, $x(t)$ is the input signal, and $h_{\mathrm{j}}$ represents the gain of the $j^{\text {th }}$ photoreceiver element. Fig. 3 also illustrates an example for 4-bit header ' 1101 ' and shows the output signal from the combiner when the gain profile matches the header sequence.

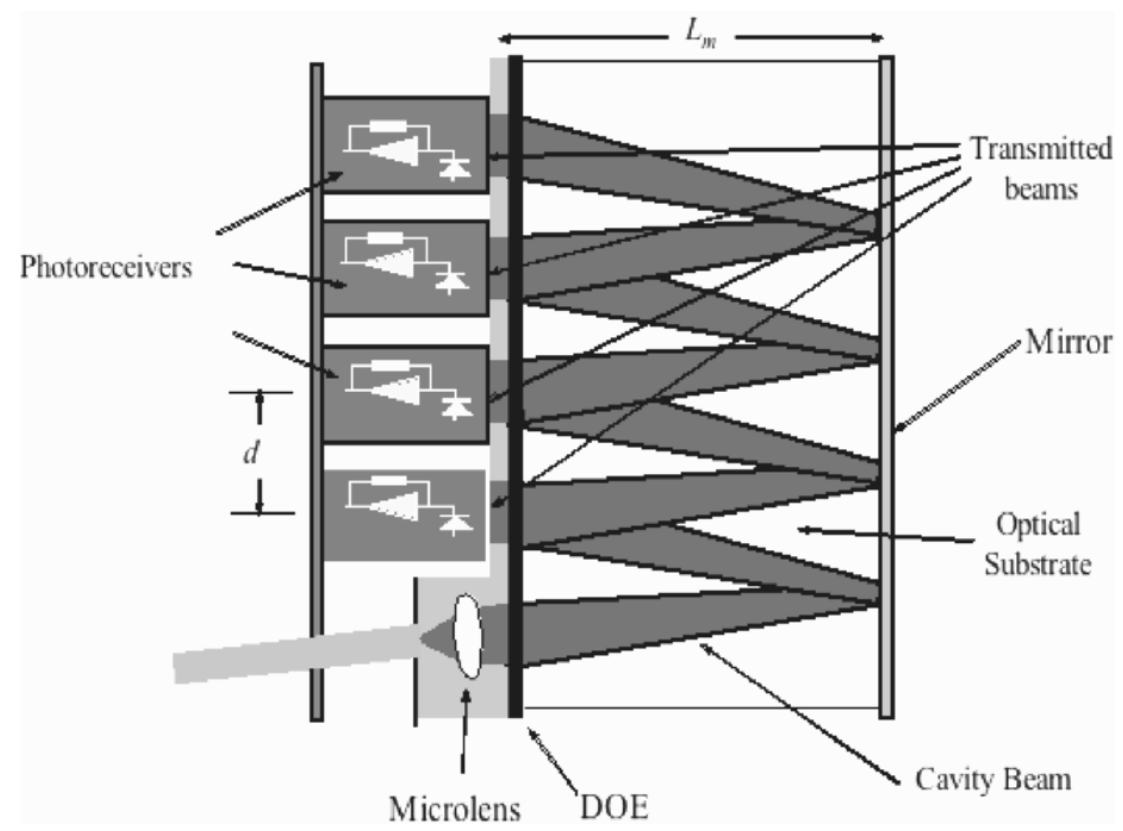

Fig. 4. Interface between the photoreceiver chip and the DOE

\section{Experimental setup}

To demonstrate the concept of the proposed header recognition architecture, we constructed a 4-bit experiment shown in Fig. 5. An HP 70841B pattern generator was used to generate a 4-bit packet at $16.1 \mathrm{Mbit} / \mathrm{s}$, which intensity modulates a $1550 \mathrm{~nm}$ optical carrier generated by an Agilent 8164A laser source, through a Mach-Zhender electro-optic modulator. The intensity modulated optical signal is equally split into four output fibre delay ports, each port delays the signal 1 bit-time longer than the previous branch using single-mode fibre line delays. A photoreceiver array, which integrates four discrete photodetectors, four variable-gain transimpedance amplifiers, and an RF combiner, were designed to provide arbitrary gain patterns by simply switching the amplifiers gains between "HIGH" and "LOW" levels. 


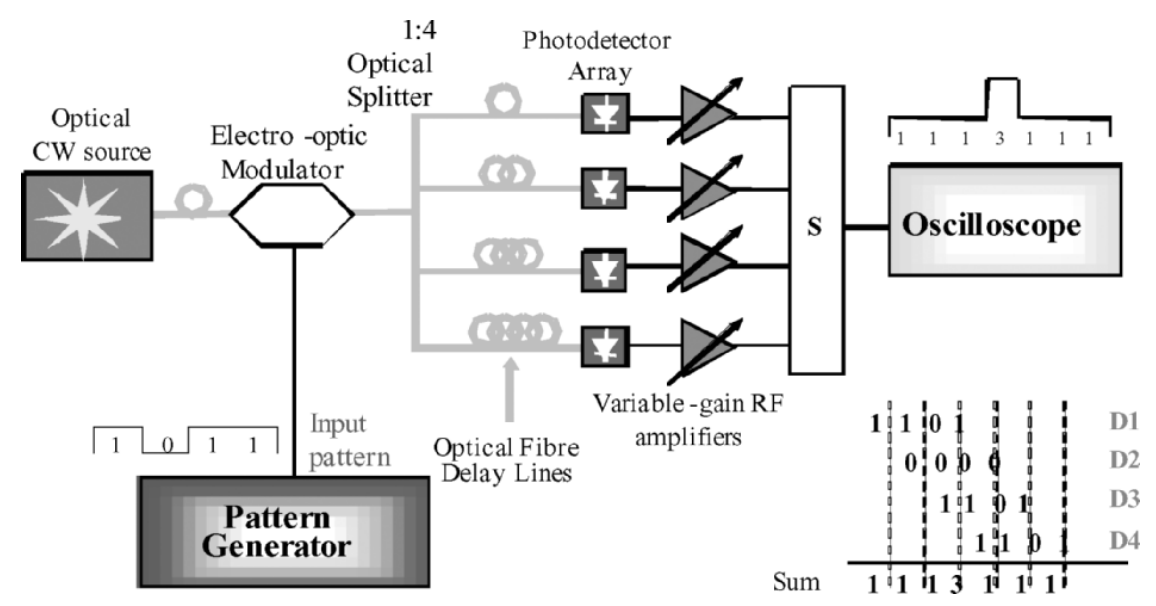

Fig. 5. Correlator experimental setup

The correlator is first configured to match a header pattern of "1011". This is accomplished by reducing the gain of the second transimpedance amplifier to a low value, corresponding to the " 0 " state, and increasing the gains of the other amplifiers to high levels, which correspond to " 1 " states. The output electrical signal from the $\mathrm{RF}$ combiner is monitored by an HP 54120A $20 \mathrm{GHz}$ digital oscilloscope.

Fig. 6 shows the measured and simulated output waveforms for the 1011 optical header. A good agreement between theory and experiment is seen, and a stable output autocorrelation is demonstrated with no optical interference. A symmetrical autocorrelation function with high amplitude (spike) is clearly displayed when the input pattern matches the amplifier gains.

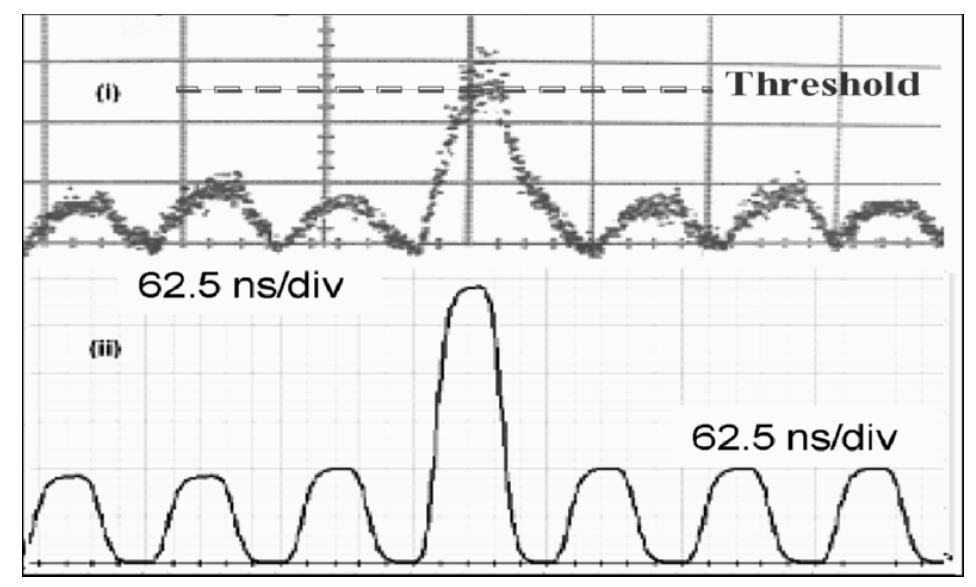

Fig. 6. i) Experimental results matched pattern, ii) simulation result matched pattern 
The correlator is also configured to match a header pattern of " 1110 ". This is accomplished by reducing the gain of the fourth transimpedance amplifier to a low value, corresponding to the " 0 " state, and increasing the gains of the other amplifiers to high levels, which correspond to " 1 " states. The output electrical signal from the RF combiner is monitored by an HP 54120A $20 \mathrm{GHz}$ digital oscilloscope.

Fig. 7 shows the measured and simulated output waveforms for the 1110 optical header. Again, high amplitude (spike) is seen when the input pattern matches the amplifier gains, and a good agreement between theory and experiment is demonstrated, with no optical interference displayed. .

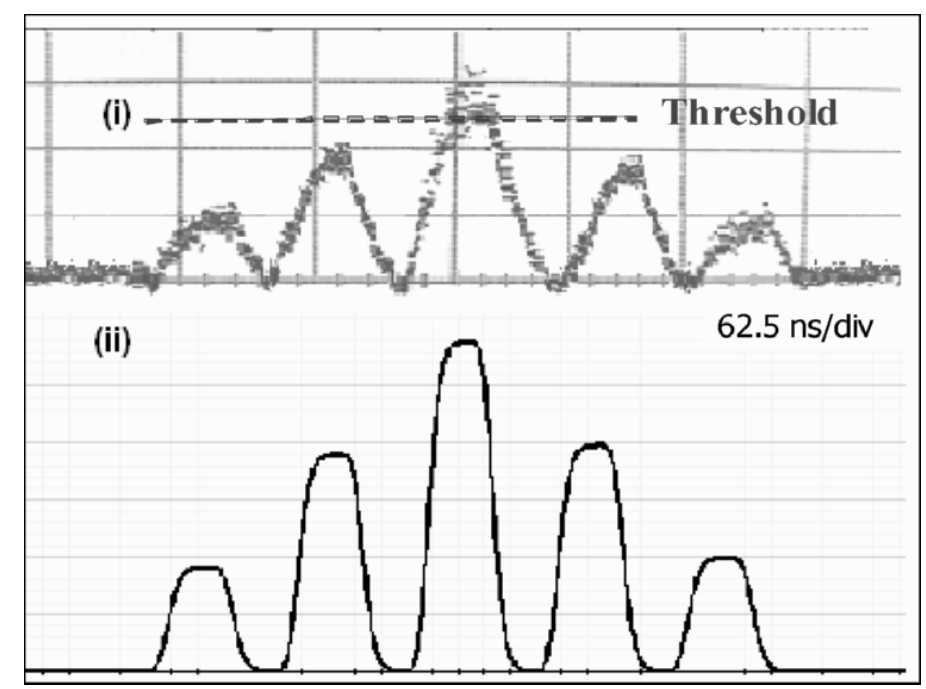

Fig. 7. Correlator match output waveform for 1110 header bit stream, i) Experiment, ii) Simulation

Note that the correlator configured for 1011 will also produce a level " 3 " peak that is above the threshold at time for a " 1111 " input, which is not the desired bit pattern as shown in Fig. 8. Error-free header recognition can be accomplished by adding a second correlator that is configured in complement to the first correlator which produces a ZERO at the centre of the output correlation when the pattern matches the gain profile and ONE otherwise [3], as shown in Fig. 9.

While the optical correlator enables on-the-fly processing of incoming packets, there are some issues associated with packets processing without converting them to electronics for header processing and updating. For example, the IP header's time-tolive (TTL) field is not decremented and the header checksum is not recomputed, whereas protocol requires that both of these operations occur at each network hop. One potential solution to this problem is to revise the protocol to allow for packets to traverse a small number of core network hops without Optical-to-Electrical (O/E) conversion and then update these fields once they reach a fully electronic router at the core edge [27]. Optical signal-processing techniques have been developed as an alternative approach to directly process these fields in the optical domain where 
instead of using binary fields within the headers a number of optical pulses are used, which correspond to the value of the TTL [28-30].

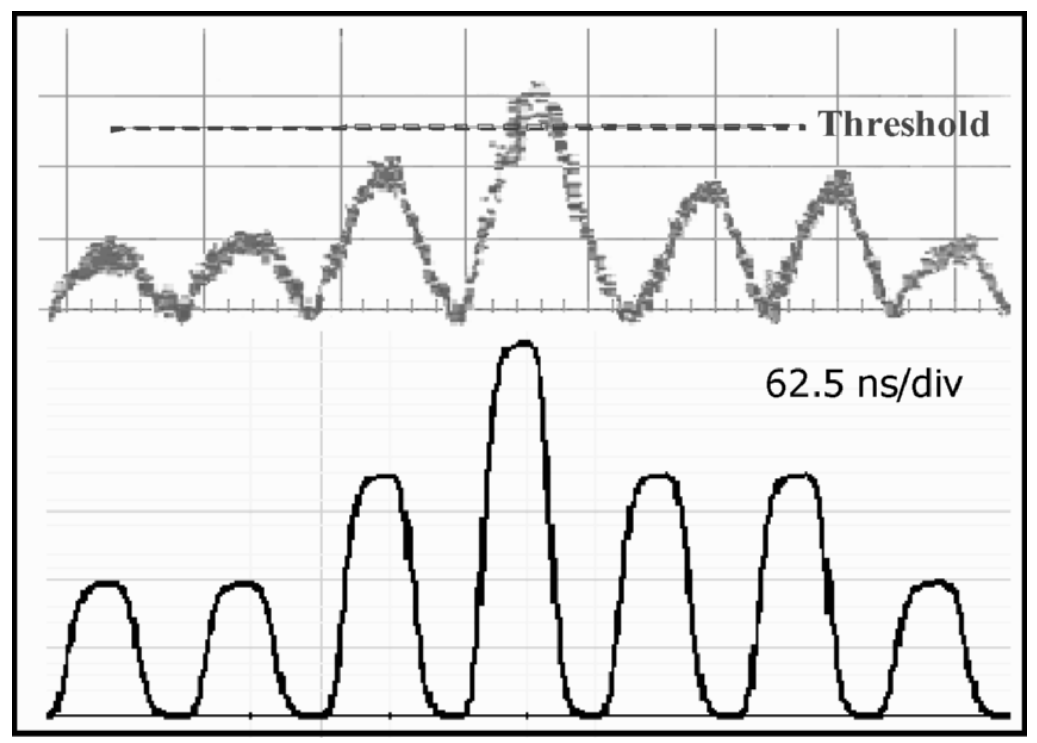

Fig. 8. Output correlation waveform showing a spike in a specific case of header-gain mismatch
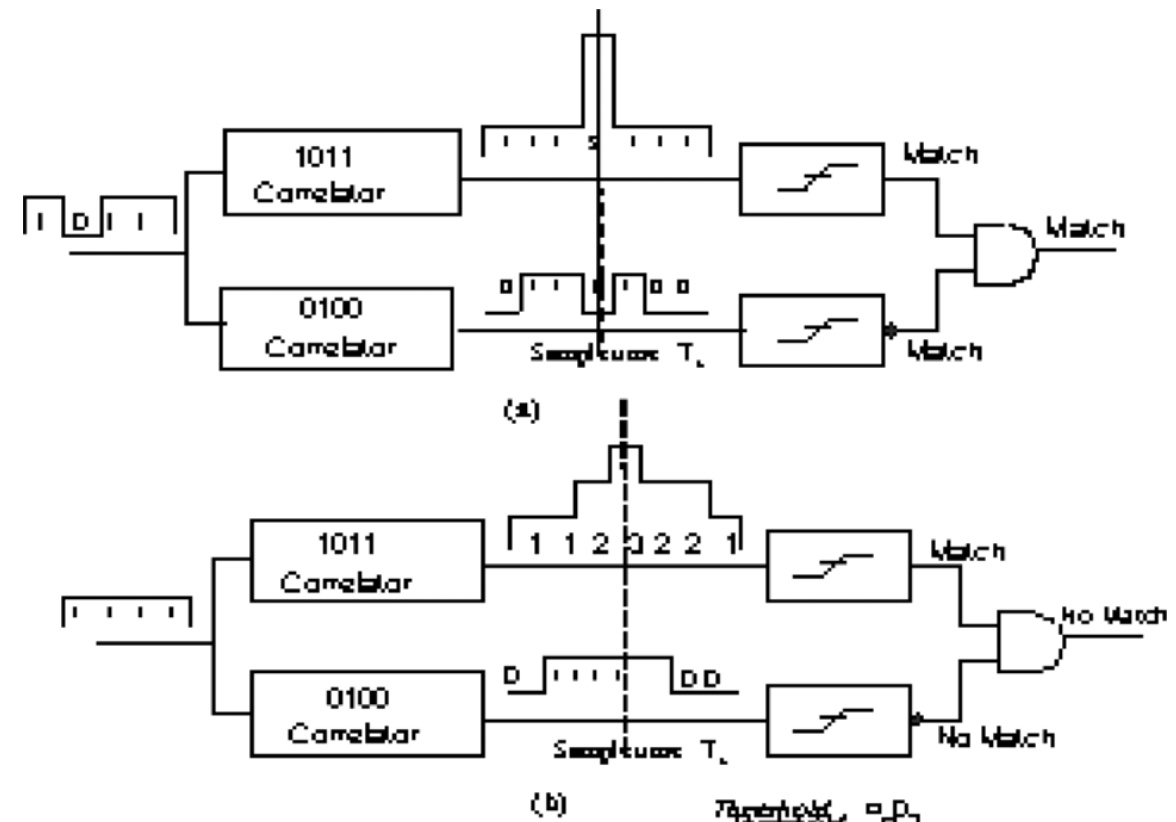

Fig. 9. Complement correlator configuration for error-free header recognition 


\section{Conclusion}

In this paper, we presented a novel MicroPhotonic header recognition architecture that is compact, simple to implement, and scalable to higher bit rates. A 4-bit correlator module was constructed, using optical fibre delay lines in conjunction with discrete photoreceiver elements, and used to experimentally demonstrate the recognition of packet headers at 16.1 Mbps. The proposed architecture has applications in optical networks and photonic RF signal processing.

\section{References}

1. W. Huang, and I. Andonovic, Coherent Optical Pulse CDMA Systems Based On Coherent Correlation Detection, IEEE J. Trans. Commun. 47 (2), 261-271 (1999).

2. R. Clavero, J.M. Martinez, F. Ramos, and J. Marti, All-Optical Packet Routing Scheme for Optical Label-Swapping Networks, OSA Opt. Express 12 (18), 43264332 (2004).

3. M.C. Hauer, J.E. McGeehan, S. Kumar, J.D. Touch, J. Bannister, E.R. Lyons, C.H. Lin, A.A. Au, H.P. Lee, D.S. Starodubov, and A.E. Willner, Optically Assisted Internet Routing Using Arrays of Novel Dynamically Reconfigurable FBG-Based Correlators, J. Lightwave Technol. 21(11), 2765-2778 (2003).

4. D.J Blumenthal, Optical Packet Switching, in Proc. IEEE/LEOS 2, 910-912 (2004).

5. P. Parolari, L. Marazzi, D. Rossetti, G. Maier, and M. Martinelli, Coherent-toIncoherent Light Conversion for Optical Correlators, J. Lightwave Technol. 18(9), $1284-1288$ (2000).

6. J.D. Shin, Y.M. Jeon, and C.S. Kang, Fiber-Optic Matched Filters With Metal Films Deposited on Fiber Delay-Line Ends for Optical Packet Address Detection, IEEE Photon. Tech. Lett. 8(7), 941-943 (1996).

7. J.D. Shin, M.Y. Jeon, E.Lee, $10 \mathrm{~Gb} / \mathrm{s}$ Optical Packet Header Recognition Using A New Fiber-Optic Matched Filter, in Proc. CLEO/Pacific Rim'95, 8 (1995).

8. X.A. Shen, Y.S. Bai, R. Kachru, Demonstration of Optical ATM Header Decoding By Spectroholographic Filtering, in Proc. CLEO '96, 222-223 (1996).

9. D. Cotter, J.K. Lucek, M. Shabeer, K. Smith, D.C. Roger, D. Nesset, and P. Gunning, Self-Routing of $100 \mathrm{Gbit} / \mathrm{S}$ Packets Using 6 Bit Keyword Address Recognition, Electronics Lett. 31(17), 1475-1476 (1995). 
10. F. Forghieri, A. Bononi, P.R. Prucnal, Novel Packet Architecture for All-Optical Ultrafast Packet-Switching Networks, IEEE Electronics lett. 28(25), 22892291(1992).

11. I. Glesk, J.P. Solokoff, and P.R. Prucnal, All-Optical Address Recognition And Self-Routing In A 250 Gbit/S Packet-Switched Network, Electronics Lett. 30(16), 1322-1323 (1994).

12. D.F. Geraghty, J. Castro, B. West, and S. Honkanen, All-Optical Packet Header Recognition Integrated Optic Chip, in Proc. IEEE-LEOS'03 2, $752-753$ (2003).

13. K. Chan, F. Tong, C.K. Chan, L.K. Chen, and W. hung, An All-Optical Packet Header Recognition Scheme for Self-Routing Packet Networks, in Proc. OFC, 284285 (2002).

14. R. Takahashi, and H. Suzuki, 1-Tb/s 16-b All-Optical Serial-To-Parallel Conversion Using a Surface-Reflection Optical Switch, IEEE photon. Technol. Lett. 15(2), 287-289, 2003.

15. R. Takahashi, T. Nakahara, H. Takenouchi, and H. Suzuki, 40-Gbit/s Label Recognition and 1x4 Self-Routing Using Self-Serial-to-Parallel Conversion, IEEE Photon. Technol. Lett. 16(2), 692-694 (2004).

16. O. Boyraz, Y. Han, A. Nuruzzaman, and B. Jalali, Time Stretch Optical Header Recognition, in Proc. IEEE-LEOS'03 2, 543-544 (2003).

17. R. Zheng, M. Aljada, Z. Wang, and K. Alameh, An Opto-VLSI Correlator for Optical Header Recognition, in proc. OFC'06, paper OThS4, (2006).

18. D.B. Hunter and R.A. Minasian, Programmable High-Speed Optical Code Recognition Using Fibre Bragg Grating Arrays, Electronics Lett. 35(5), 412-414 (1999).

19. J.E. Mcgeehan, M.C. Hauer, And A.E. Willner, Optical Header Recognition Using Fiber Bragg Grating Correlators, IEEE LEOS Newsletter 16(4), 29-32 (2002).

20. M.C. Cardakli, S. Lee, A.E. Willner, V. Grubsky, D. Starodubov, and J. Feinberg, All-Optical Packet Header Recognition And Switching In A Reconfigurable Network Using Fiber Bragg Grating For Time-To-Wavelength Mapping And Decoding, in Proc. OFC/IOOC'99 3, 171- 173 (1999).

21. M.C. Cardakli, S. Lee, A.E. Willner, V. Grubsky, D. Starodubov, and J. Feinberg, Reconfigurable Optical Packet Header Recognition and Routing Using Time-To-Wavelength Mapping and Tunable Fiber Bragg Gratings for Correlation Decoding, IEEE Photon. Technol. Lett. 12(5), 552-554 (2000). 
22. D. Gurkan, M.C. Hauer, A.B. Sahin, Z. Pan, S. Lee, and A.E. Willner, Demonstration Of Multi-Wave All-Optical Header Recognition Using PPLN and Optical Correletors, in Proc. 27 ECOC'01 3, 312-313 (2001).

23. A.E. Willner, All-Optical Packet-Header-Recognition Techniques, in proc. IEEE/LEOS 1, 47-48 (2002).

24. M.C. Cardakli, D. Gurkan, S.A. Havstad, and A.E. Willner, Variable-Bit-Rate Header Recognition for Reconfigurable Networks Using Tunable Fiber-BraggGratings As Optical Correletor, in Proc. OFC 1, 213 -215 (2000).

25. A.E. Willner, All-Optical Signal Processing For Implementing Network Switching Function, in Proc. IEEE/LEOS'02, TuC1-9-TuC1-10, (2002).

26. J.E. McGeehan, M.C. Hauer, A.B. Sahin, and A.E. Willner, MultiwavelengthChannel Header Recognition for Reconfigurable WDM Networks Using Optical Correlators Based on Sampled Fiber Bragg Gratings, IEEE Photon. Technol. Lett. 15(10), 1464-1466 (2003).

27. G.G. Finn, S. Hotz, and C. Rogers, Method And Networking Interface Logic For Providing Embedded Checksums, U.S. Patent 5 826 032, (1998).

28. J.E. McGeehan, S. Kumar, J. Bannister, J. Touch, and A.E. Willner, Optical Time-To-Live Decrementing And Subsequent Dropping Of An Optical Packet, in Proc.OFC'03 2, $798-801$ (2003).

29. J.E. McGeehan, S. Kumar, D. Gurkan, S.M.R.M. Nezam, A.E Willner, K.R. Parameswaran, M.M. Fejer, J. Bannister, J.D. Touch, All-Optical Decrementing of a Packet's Time-to-Live (TTL) Field and Subsequent Dropping of a ZeroTTL Packet, J. Lightwave Technol. 21(11), 2746-2752 (2003).

30. W. Hung, K. Chan, L.K. Chen, C.K. Chan, F. Tong, A Routing Loop Control Scheme In Optical Layer For Optical Packet Networks, in Proc. OFC, 770-771 (2002). 